22 January 2009

\title{
Automatic Whistler Detection: Operational Results from New Zealand
}

\author{
Craig J. Rodger
}

Department of Physics, University of Otago, Dunedin, New Zealand

János Lichtenberger

Space Research Group, Eötvös University, Budapest, Hungary

Gregory McDowell and Neil R. Thomson

Department of Physics, University of Otago, Dunedin, New Zealand

Abstract. Lightning-generated "Whistlers", the strongly dispersed radio wave pulses that have propagated along the Earth's magnetic field from one hemisphere of the Earth to the other, have long been regarded as inexpensive and effective tools for plasmasphere diagnosis. The Eötvös University Automatic Whistler Detector (AWD) system has been operating in Dunedin, New Zealand since mid-May 2005. Here we report on the first 530 days of near-continuous AWD operation. In this time period the AWD system detected 92,528 individual whistler events containing 236,019 whistler traces. This equates to a whistler event rate of 0.12 per minute, and a whistler trace rate of 0.31 per minute. Despite the conjugate lightning rate for Dunedin being a factor of $\sim 1500$ lower than that for Hungary, the AWD-reported whistler rate from Dunedin was only $\sim 3$ times lower. Dunedin whistler rates are high, hundreds of times higher than estimated from the conjugate lightning activity, showing that conjugate lightning activity levels are not a good predictor of whistler rates. Dunedin-observed whistlers are most common during the day time, in stark contrast with earlier findings and general expectations. We suggest that North American lightning may be the principal source of Dunedin-whistlers.

The Dunedin-based AWD has detected a large number of whistlers over a wide range of $L$ shells, with a sufficiently small false trigger rate $(\sim 58 \%)$ to allow rapid processing of the data. 
Dunedin observations may soon provide a valuable near-continuous plasmaspheric measurement stream, after the planned upgrade of the existing AWD to an automatic analyzer and AWD system.

\section{Introduction}

Whistlers are electromagnetic phenomena produced by the propagation of the radio energy from lightning-generated sferics through the ionospheric and magnetospheric plasma [Storey, 1953]. Whistlers are identified as audio-frequency radio signals that usually begin at high frequencies (on the order of $10 \mathrm{kHz}$ ) and typically fall in frequency to $\sim 1 \mathrm{kHz}$ in about $1 \mathrm{~s}$. Some whistlers are pure descending tones while others are described as "swishy", being less spectrally pure. Whistlers observed on the ground have propagated in field-aligned ionisation irregularities, termed "whistler ducts", which extend between the conjugate hemispheres. Whistlers are often observed by ground-based receivers to occur in groups, one example being "multi-trace" or "mixed-path" whistlers caused by the reception of multiple whistler traces produced by the same lightning discharge but travelling through different ducts. Another example is an "echo-train", where the whistler-wave bounces back and forth between the conjugate hemispheres, leading to odd numbered hops in the hemisphere conjugate to the source, and even numbered in the source hemisphere. It is generally accepted that ground-based whistler observation is more common during nighttime [Helliwell, pg. 144, 1965] due to decreased ionospheric absorption. A historical overview of whistler research has been presented by Al'pert [1980].

The fundamental nature of the received whistler depends upon on its propagation through the magnetosphere [see the review by Sazhin et al., 1992]. The dispersion and group delay are primarily dependent upon the cold plasma electron density and the McIlwain L-shell [McIlwain, 1961] of the whistler duct. Thus observation of whistlers from a ground-based station provides a 
technique for monitoring cold plasma densities, particularly inside the plasmasphere where whistlers are most common, and have long been regarded as inexpensive and effective tools for plasmasphere diagnostic [Park, 1972]. Plasmaspheric electron densities are an important parameter for describing the condition of the near-Earth plasma and the waves that propagate through this plasma. They are a fundamental "input" parameter required to calculate wave-particle interactions which govern the acceleration and loss of particles trapped in the Van Allen radiation belts [e.g. Lyons et al., 1972]. Plasmaspheric electron densities strongly determine the resonant energy for energetic electrons resonating with VLF waves [e.g., Chang and Inan, 1983], or EMIC waves [Meredith et al., 2003]. In addition, the path by which non-ducted whistler mode waves propagate through the plasmasphere is also strongly influenced by Plasmaspheric electron densities [e.g., Starks et al., 2008].

In many parts of the world whistlers are fairly common. Broadband very low frequency (VLF) surveys at multiple stations in the International Geophysical Year found whistler rates ranging from essentially zero up to $\sim 9$ whistlers per minute [Helliwell, Fig. 4-39, 1965]. However, it is not practical to manually search continuously recorded VLF data for whistler events as a source of regularly measured plasmaspheric parameters; while realistic for case studies, the time commitments make this impractical otherwise. For this reason the Eötvös University "Automatic Whistler Detector" (AWD) system [Lichtenberger et al., 2008] has been developed as a step towards producing automatic and near continuous ground-based plasmaspheric measurements. The AWD system was first deployed in Tihany, Hungary $\left(46.89^{\circ} \mathrm{N}, 17.89^{\circ} \mathrm{E}, L=1.81\right)$ and identified 252,000 whistler traces over a two-year period [Collier et al., 2006], i.e., a whistler trace rate of $0.35 \mathrm{~min}^{-1}$. The seasonal occurrence of Tihany-observed whistlers was found to be strongly dependent upon lightning activity in the southern African conjugate region. Whistler observations peaked in the same month that lightning activity peaked, although the diurnal variation in whistler occurrence peaked in the nighttime and was principally determined by 
ionospheric absorption, rather than the late afternoon-early evening when conjugate lightning activity peaked.

The relatively high whistler rate observed from Tihany suggests it would be well suited to provide ground-based plasmaspheric measurements, with a relatively high time resolution. This can be understood in terms of the high lightning activity in the southern African conjugate region, producing a strong whistler source. Tihany has some limitations, however, with very few multipath whistlers, and $80 \%$ of the whistlers falling in the range $L=2-2.8$ [Tarcsai et al., 1988]. In order to improve the database of plasmaspheric measurements, multiple AWD-receiving stations will be deployed in the future over a wide range of longitudes to sample in Local Time (LT) and $L$. Few of the practical locations where these systems can be deployed have conjugate lightning activity levels which are comparable to southern Africa, which may affect the quantity of plasmaspheric measurements such a network could produce. In this study we report on whistler observations from the AWD station on the opposite side of the Earth, in a region where lightning activity in the conjugate hemisphere is comparatively low. Our goal is to examine the effectiveness of a Dunedin-based whistler system, such that it may soon provide regular and semiautomatic measurements of plasmaspheric densities to the wider scientific community.

\section{Lightning-Whistler Connections: Identifying an apparent paradox}

Satellite observations now allow some confidence in the average geographical distribution of total lightning activity and global flash rate. Five years of Optical Transient Detector (OTD) observations have been combined to produce typical lightning density distributions [Christian et al., 2003]. For one-hop whistlers, one would expect the appropriate lightning source population to be that in the geomagnetic conjugate hemisphere. For this reason we have transformed the global geographical maps of total lightning activity (in units of flashes $\mathrm{km}^{-2} \mathrm{yr}^{-1}$ ) taken from the OTD Low Resolution Full Climatology dataset [Christian et al., Fig. 4, 2003] into magnetic 
coordinates and mirrored them about the geomagnetic equator. Figure 1 shows the annual average global total lightning activity in Corrected GeoMagnetic (CGM) coordinates based on the Definite/International Geomagnetic Reference Field (DGRF/IGRF) for 2003 at $100 \mathrm{~km}$ altitude, using the GEOPACK software routines. To aid the eye, this figure shows the geographical coastlines also translated into CGM coordinates. The upper panel in Figure 1 shows southern hemisphere lightning mirrored into the northern hemisphere, representing the expected conjugate lightning population producing one-hop northern hemisphere whistlers. The lower panel shows northern hemisphere lightning mirrored into the southern hemisphere. In both cases dots mark AWD receiving locations in New Zealand and Hungary, and their geomagnetic conjugates. Lightning activity has been suppressed on this plot for very low latitudes $\left(<5^{\circ}\right)$, where the GEOPACK calculation is not reliable. Note that a map of OTD lightning activity in geomagnetic coordinates without the mirroring transformation may be found in Rodger et al. [2005].

The upper panel of Figure 1 confirms that Tihany is well located for whistler measurements, assuming that the lightning levels in the conjugate region strongly influence the whistler rates. Few other northern hemisphere locations are as well suited, given that ground-based whistler receiving stations at low geomagnetic latitudes tend to report very low occurrence rates [Helliwell et al., Fig 4-40, 1967]. Based on conjugate lightning activity, Korea and Japan would provide potential high-whistler rate sites for AWD deployment. The lower panel of Figure 1 presents the one-hop whistler source population for southern hemisphere receiving locations. The highest conjugate lightning rates are to the west of the Antarctic Peninsula, consistent with the high whistler occurrence rates reported there [e.g., Burgess and Inan, 1993] including some subionospheric propagation of the whistler from the duct exit point. Few other southern hemisphere non-ocean locations are located near high conjugate lightning levels. As an extreme example, the conjugate lightning levels near New Zealand are $\sim 1500$ times lower than those 
around Hungary, implying an average whistler trace rate of $\sim 2.3 \times 10^{-4} \mathrm{~min}^{-1}$, i.e. less than one whistler received per day. Given that no automatic system is likely to be $100 \%$ efficient, this estimated whistler rate seems to imply that whistlers are unlikely to be useful as a plasmaspheric probe for New Zealand longitudes. However, historic reports of whistler activity in New Zealand suggest that the rates estimated above are many orders of magnitude too low. For example, observations from New Zealand over 25 months between January 1958 and October 1960 produced an average whistler rate of more than $2 \mathrm{~min}^{-1}$, roughly similar to the whistler rates in central Europe [Helliwell, 1965].

The historic whistler studies from New Zealand suggest that it is well suited for such monitoring of the plasmasphere, in contrast with expectations from the conjugate lightning levels alone. On this basis we deployed an AWD system to New Zealand to examine this apparent paradox.

\section{Automatic Whistler Detector: Experimental Setup}

The operation of the AWD has been previously described by Collier et al. [2006]. A detailed description of the system operation and algorithm development can be found in Lichtenberger et al. [2008]. We briefly summarize its operation here to assist the reader, and describe the specifics of the operation in New Zealand. The incoming VLF datastream is sampled at a rate of 44.1 kHz using a 16-bit soundcard in a standard personal computer. The datastream is taken from the magnetic North-South loop antenna sited at Swampy Summit, Dunedin, New Zealand (45 $\left.47^{\prime} \mathrm{S}, 170^{\circ} 28^{\prime} \mathrm{E}, L=2.7\right)$. While the operational system can independently sample from the datastreams provided from both the NS and EW loops, there were high man-made noise levels in the EW loop observations at the time of installation, and thus we only trigger off the NS loop. While this will degrade the detection of whistlers propagating from the east or west of Dunedin, this should only affect a small subset of events, as only a small range of east-west propagation 
angles will have a very small component in the NS antenna. The Eötvös University-developed whistler detection algorithm automatically identifies whistler traces from the incoming data stream relying on 2D image correlation to identify the whistler in the wideband VLF data stream [Lichtenberger et al., 2008]. The traces are correlated against a pattern made from an approximate model whistler equation [Bernard, 1973], covering a range of dispersion values from $D_{0}=40-100 \mathrm{~Hz}^{1 / 2}$. The detector is tuned for one-hop whistlers by providing it with appropriate dispersion $\left(D_{0}\right)$ and nose-frequency $\left(F_{n}\right)$ pairs, i.e. combinations of physically appropriate values of $D_{0}$ and $F_{n}$, which in a real whistler are linked and constrained in range. Operationally we found that Dunedin receives many low latitude whistlers (spanning roughly $\left.D_{0}=50-60 \mathrm{~Hz}^{1 / 2}, F_{n}=20-50 \mathrm{kHz}\right)$, as well as many mid- to high-latitude whistlers $\left(D_{0}=70-90 \mathrm{~Hz}^{1 / 2}\right.$ and $F_{n}=5-10 \mathrm{kHz}$ ), and thus a higher upper dispersion limit must be set than was used in South Africa or Tihany, where the detector used $20-80 \mathrm{~Hz}^{1 / 2}$ [Collier et al., 2006]. The Dunedin whistler observations are different from those seen at Tihany, where the whistlers received are from lower-latitude paths. In practice, multi-hop whistlers have increased dispersions (e.g., a 2hop whistler has a doubled dispersion) but with an unchanged nose frequency. Thus multi-hop whistlers are significantly different patterns for image correlation (as described by Lichtenberger et al. [2008]), and the AWD algorithm is essentially insensitive to these events given the range of dispersions set for the Dunedin AWD instrument. This algorithm applies other techniques [Lichtenberger et al., 2008] to remove such unwanted effects as sferics, powerline harmonics and VLF transmitters used for military and navigational transmissions (e.g., the Alpha network [Barr et al., 2000]).

In this paper we report on the analysis of the first 530 days of AWD system operation at Dunedin, spanning the period from 20 May 2005 - 31 October 2006. The developmental philosophy of the whistler detection algorithm was to "over trigger", i.e., to attempt to capture all possible whistlers but accept some events will be captured which do not represent whistlers. The 
whistler detector output is then manually examined "by eye" to determine if the $4 \mathrm{~s}$ captured record contains a whistler. Over the first 530 days of operation the AWD system captured 147,339 records, of which 61,448 contained whistlers, i.e., a system accuracy of $\sim 42 \%$, and thus a false trigger rate of $\sim 58 \%$. This is at the lower end of the $50-80 \%$ false detection rate reported by Lichtenber et al. [2008] on the basis of 3 years of observations at different locations. While the Lichtenberger study indicated that many of their false detections were caused by local noise and sferic "remnants", a number of the Dunedin false triggers were due to other VLF emissions (e.g., chorus, hooks, etc), which may provide some explanation for the lower system accuracy value. As VLF emissions can contain curved elements which in a spectrogram-view resemble parts of a whistler (see the examples in Chapter 7 of Helliwell [1965]), the AWD-algorithm may trigger on these events, which at this time are manually discarded through a manual "by eye" examination. A discussion on the false-triggering of AWD-systems due to the most common situation of sferic remnants is given in Section 2.2.5 of Lichtenber et al. [2008]. While the false trigger rate is $>50 \%$, and hence more than half of the Dunedin AWD output files do not contain a whistler, the false trigger rate is sufficiently small to allow rapid processing of the output data, and the generation of a whistler database. Even with the false trigger level, the time requirements are significantly more than an order of magnitude lower, when contrasted with a manual search of continuous wideband VLF data. Some of these files contained multiple whistler events, and some of these events included multiple traces. An example of a rather spectacular whistler event captured by the Dunedin-based AWD on 4 February 2006 is shown in Figure 2.

\section{Results}

\subsection{Total Numbers, Traces, and Rates}

The 61,448 whistler-containing files were processed as outlined in Collier et al. [2006] to extract the number of "events" and "traces" within a file. Here we define an event as being a 
whistler generated by a single lightning flash, which may lead to a single-trace whistler or a multiple trace whistler group if the lightning energy has travelled along multiple paths through the plasmasphere. However, in order to implement this definition in the processing algorithm, an approach needs to be taken which generally separates the multiple trace events where the event consists of widely spaced traces, albeit generated by a single lightning discharge. An empirical approach was taken to automatically determine the number of traces in a multipath grouping, through tests developed on observed data, which has been described in more detail in Lichtenberger et al. [2008], but is summarized here. A single whistler trace was found to produce a correlation output narrower than $\sim 40-50 \mathrm{~ms}$, while multi-path traces usually produce much wider correlation outputs, up to a few hundred milliseconds. Thus an output wider than $45 \mathrm{~ms}$ is treated as a multipath group of whistler traces and the number of traces inside estimated using and average width of $30 \mathrm{~ms}$. In practice this will underestimate the number of multi-path groups and the number of traces in a multi-path group, because there can be gaps within a group with missing or low energy traces. In this case such a single group will be classified as two separate groups with a smaller number of traces overall. Future AWD processing algorithms are to have improved trace counting approaches, planned after the automatic analysis of AWDwhistlers [Lichtenberger et al., 2008] has been made operational.

After processing we found that the AWD captured 92,528 individual whistler events over the period 20 May 2005 - 31 October 2006, equating to a whistler event rate of 0.12 per minute. Clearly, lightning activity in the conjugate region is not necessarily a good indication of the expected whistler rate, as this is 500 times higher than predicted from lightning rates. Multi-trace whistlers, following numerous distinct paths through the magnetosphere, are rare at low $L$, and the vast majority of the whistlers observed by the Tihany AWD are single-trace events. However, this is not true for the Dunedin AWD; there were 236,019 whistler traces present in the Dunedin-detected whistler events. Figure 3 shows the variation in the number of whistler-traces 
per event in the Dunedin data. About $58 \%$ of the Dunedin observed whistlers are made of a single trace, i.e. roughly $42 \%$ are multi-trace. The multi-trace whistlers contain between $2-33$ traces, with the average number of traces for all Dunedin whistler events being $\sim 2.6$ traces/event. Note that the $y$-axis of this figure has been presented with a logarithmic scale, with only 19 whistlers included in this plot with more than 25 traces. It is likely that multi-trace whistlers will provide some of the most interesting plasmaspheric electron density data, as they simultaneously sample a number of different $L$-value paths. As noted above, $\sim 42 \%$ of Dunedin whistler events are multi-trace, with an average of $\sim 4.7$ traces present in the multi-trace whistlers, i.e., excluding single trace whistlers. Note that the variation in the number of whistler-traces per event shown in Figure 3 has a strong exponential relationship, particularly in the range from 4-22 traces. Clearly, some physical process must be leading to such a strong relationship. However, it is not clear what this process might be, and one can speculate as to multiple different drivers which could influence this distribution. For example, one can speculate that the observed relationship may be due to the known tendency for duct output power to generally decrease with increasing L [Smith et al., 2001], combined with increasing subionospheric attenuation experienced for signals from higher-L ducts on their propagation to Dunedin. Alternatively, the observed relationship might reflect the distributions of ducts and sub-ducts (sometimes referred to as ductlets) in the plasmasphere.

Collier et al. [2006] found that the whistler rate at Tihany was 0.35 whistlers per minute, with the whistler event rate and whistler trace rate being essentially identical. However, in the Dunedin data the whistler event rate is 0.12 per minute, while on average there were 0.31 whistler traces per minute. We note that the whistler rate at Dunedin is very roughly similar to that observed at Tihany, despite the vastly different conjugate lightning flash rates. It appears that the observed whistler rates from Dunedin are sufficiently high to provide a regular monitoring tool for plasmaspheric densities, as will be discussed in a later section. High 
sampling rates due to high whistler rates may allow the detection and characterization of plasmaspheric plumes [Borovsky and Denton, 2008], which have been linked to dropouts in of relativistic electron fluxes at geosynchronous orbit during geomagnetic storms [Borovsky and Steinberg, 2006].

Depending on the intended application, one or other of the event and trace rates may be more appropriate. For example, the event rate will be the most relevant for the purposes of determining the typical rate at which whistler plasmaspheric measurements might be provided. In contrast, the whistler trace rate will be important for estimating the rate at which a measurement with additional $L$-variation is possible. Note, of course, that these whistler rates are the AWDdetermined detection rates. We do not expect that the detection efficiency of the AWD system is $100 \%$, or that all AWD-detected whistlers can be meaningfully scaled to plasmaspheric electron densities. An examination of these factors will be left to future studies.

\subsection{Seasonal Variation}

It is well known that the reception of whistlers at ground-based stations depends upon multiple factors. The summary provided in the classic text by Helliwell [1965] demonstrates the seasonal dependence produced by thunderstorm activity and noise levels (Fig 4-38) and the availability of whistler ducts (pg. 149). Confirming elements of this picture, Collier et al. [2006] also demonstrated that the connection between whistlers and lightning is strongly modified by ionospheric absorption. Our AWD-station in Dunedin is unusual in that it is located well away from strong lightning activity, in either hemisphere. The Dunedin AWD observations allow us to test how general these conclusions are.

Figure 4 shows the daily variation in Dunedin observed whistlers, presented as a daily whistler trace rate. As previously reported, the observed whistler rate is highly variable; in the $\sim 1.5$ years of AWD operation reported here, the number of whistler traces captured on a given day has 
varied from 0 to 11,828 . On the extreme high activity day, the average whistler trace rate across the day was $\sim 8.2 \mathrm{~min}^{-1}$, more than 25 times larger than the long-term average. Only 73 days (14\%) had less than 10 whistler traces captured by the AWD. We note, however, that high whistler rates are not a sufficient condition for high time resolution plasmaspheric measurements, as the received whistlers must also be sufficiently well defined to determine the $L$-value of their propagation path.

Also shown in Figure 4 are the daily average $K p$ and Dst values (black and red lines, respectively), to provide context as to the level of geomagnetic disturbances during the whistler observations. Clearly, there is no one-to-one correlation between Dunedin-observed whistlers and geomagnetic activity. This is particularly apparent for the period from January-June 2006, where significant geomagnetic disturbances occur, but whistler activity is low. Figure 4 suggests a weak link between Dunedin whistlers and geomagnetic activity, with the days on which there was high whistler activity occurring roughly around the times of geomagnetic disturbances. There are multiple reasons to expect such a linkage to exist, but to be complex. While lightning rates are not expected to be associated with geomagnetic disturbances, whistler amplitudes should be higher after geomagnetic storms, due to increased availability of energetic particles to cause amplification. Studies of ground-based VLF sources have shown a factor of $\sim 30$ increases in whistler mode amplitude with increasing Kp [Thomson et al., 1997].

The seasonal variation in our Dunedin AWD-observed whistler traces is shown in Figure 5. Whistler rates are relatively low in the northern hemisphere spring, but considerably larger in the northern hemisphere summer and autumn. While the very large number of whistlers observed in Dunedin is not consistent with a source near our receivers conjugate, the seasonal variation seen in Figure 5 is roughly consistent with the expected changes in lightning activity in the northern hemisphere. 


\subsection{Diurnal Variation}

It is widely accepted that the occurrence rate of ground-based whistlers peaks during the nighttime. For example the vast majority of Tihany AWD-observed whistlers, $85 \%$ and $89 \%$, were recorded when the conjugate source region and Tihany respectively were in darkness. This study suggests that the efficiency by which conjugate lightning generated whistlers was sharply modulated by the ionospheric absorption at either the receiver or conjugate hemisphere, in accordance with the conclusions of Helliwell [1965]. For Tihany the ratio between observed whistler and lightning flash rates were $\sim 3$ orders of magnitude higher during the night than day [Collier et al., Fig. 11, 2006]. At Tihany's location it was not possible to identify if absorption at the source or receiver hemisphere was more important.

Figure 6 shows the diurnal variation in the Dunedin AWD-observed whistlers. Note that the local time in Dunedin is shifted by 12 hours relative to Universal Time (i.e., UT $=$ LT -12 ). As shown in Figure 6, whistler rates in Dunedin peak at $\sim 0300$ UT, i.e. $\sim 3$ pm LT. Very high rates of whistlers are reported by the AWD from $\sim 11$ am- 6 pm LT, when both the receiver and conjugate hemisphere have daytime ionospheres, and hence the largest levels of ionospheric absorption. The lowest whistler rates are seen in Dunedin from $\sim 0800-1200$, i.e., 8 pm-midnight, in the LT period when the highest whistler rates are seen in the Tihany data (Collier et al., Fig. 8, 2006). The shape of the diurnal variation shown in Figure 6 is the opposite of that expected, but is fairly robust across the 530 day period. Roughly $70 \%$ of the Dunedin AWD whistlers occur during local daytime. The diurnal variation for the Dunedin-observed whistlers is not consistent with the expected variation in lightning activity in the region conjugate to Dunedin (peaking at 5$6 \mathrm{pm}$ LT, i.e. 0600-0700 UT) and is also not consistent with the expected variation in ionospheric absorption in this longitude sector. Our observations are in apparent disagreement with those reported from other sites across the world where whistler activity peaks shortly before sunrise [e.g., Helliwell, Fig. 4-37, 1965], i.e., during the night period for the receiver. In that 
study whistler occurrences decreased rapidly after sunrise, with very low observation rates during the receiver daytime. This contrast is particularly relevant given one of the sites used in Helliwell's monograph to demonstrate this diurnal variation was Wellington, New Zealand, which would be expected to have the same variation as Dunedin.

\subsection{Case Study: Range of $L$-shells}

For the last two decades, the primary experimental technique employed in Dunedin for measuring plasmaspheric electron densities has been the locally developed VLF "Doppler" Experiment [e.g., Thomson et al., 1997]. The Doppler instrument monitors whistler-mode signals from manmade VLF transmitters located in the northern hemisphere and detected in Dunedin. These signals have penetrated the ionosphere and propagated through the plasmasphere guided along geomagnetic field lines. Such signals allow us to monitor the nature of near-Earth space, particularly during the night. However, the Doppler instrument exists as a research tool from which multiple parameters can be derived, but with essentially no daytime coverage. In addition, the $L$-shell range monitored by the existing Doppler experiment is limited to about $L \leq 2.5$ by the half-gyro cutoff and the operating frequencies of the VLF transmitters. Despite the group's familiarity with the Doppler data, it does not appear to be well-suited to meet the goal for automated plasmaspheric measurements in a practical way.

The analysis of the Dunedin AWD observations presented above suggests that whistlers may provide sufficient measurements to be useful to the wider community. We also wish to consider the range of $L$-shells on which Dunedin-received whistlers propagate. A study undertaken of 985 whistlers observed at Tihany $(L=1.81)$ from December 1970-May 1975 found that the events spanned from $L=1.4-3.2$, with about $80 \%$ of these whistlers travelling along ducts in the range $L=2.0-2.8$, i.e. at higher $L$-value than the observing station [Tarcsai et al., 1988]. This distribution has been confirmed using AWD observations from Tihany, using 95 times more 
whistlers than the original Tarcsai study [Lichtenberger et al., 2008]. We undertook a case study of Dunedin AWD-observed whistlers during 20-25 May and 1-2 July 2005, manually scaling 372 whistlers to determine plasmaspheric electron densities and $L$-shells. On these days the whistlers probed a large range of plasmaspheric $L$-shells. In this case study time period large numbers of measurements were present spanning $L=2 \cdot 5-3.7$. Occasional rare events are also present in the case study dataset, increasing the potential $L$-shell range to $L=1.6-3.9$. While a statistical treatment of the $L$-shells probed by Dunedin whistlers will be left until automatic scaling software is developed, we note that the case study indicates that Dunedin AWD-observed whistlers span a wide range of $L$-shells, and will significantly expand the observational capacity.

\section{Discussion}

\subsection{Lightning Source Regions}

While the Dunedin-observed whistlers are one-hop, and thus from the northern hemisphere, neither the high whistler rate nor the diurnal variation supports a lightning-source located near our geomagnetic conjugate. This leads us to question the lightning source region which produces the Dunedin-observed whistlers. While the comparatively high whistler rate suggests that Dunedin whistlers could provide a valuable source of plasmaspheric densities, the question remains as to which longitude-sector of the plasmasphere the whistlers measured in Dunedin have passed through.

Clearly, conjugate lightning activity levels are not a good predictor of whistler rates, as the Dunedin and Tihany rates show no relation to the relative conjugate lightning. This could indicate that the availability of ducts is the principle driver for ground-based whistler observation, and that these are sufficiently more common near Dunedin to overcome vast differences in lightning levels. Observations from the Dunedin-based Doppler instrument provide some support for this, showing that ducts are commonly observed in this longitude 
sector [Clilverd et al., 1993]. However, long term observations from a similar instrument are required to allow a meaningful comparison with Tihany. We are unaware of studies which have considered the longitudinal variation in duct occurrence, and given that the mechanism which produces ducts is unclear, it is possible that ducts may be inhomogenously distributed in longitude. One might also speculate that the OTD lightning observations have failed to correctly detect lightning in our conjugate region. However, an examination of whistler-induced electron precipitation occurring near our Dunedin receiver and making use of 10,000 hours of subionospheric measurements showed that the observed precipitation rate agreed with the rate predicted from conjugate lightning levels [Rodger et al., 2005].

Another possibility is that whistlers observed in Dunedin are due to lightning discharges which occur far from the receivers conjugate, and hence there is not a direct relationship between conjugate lightning and observed whistlers. The Dunedin observed whistlers peak at $\sim 0300$ UT. This is approximately the time for which intense lightning activity peaks over North America [Lay et al., 2007]. Some evidence for this propagation route has already been reported. Clilverd et al. [1992] showed that transmissions from a VLF transmitter near Seattle, USA, often propagated as much $2 \mathrm{Mm}$ subionospherically before entering a duct, reaching the southern hemisphere and propagating to Dunedin a further $1 \mathrm{Mm}$. A commonly observed alternative path involved transmissions entering the duct at the transmitter longitude, but with 2-3 Mm of southern hemisphere subionospheric propagation to Dunedin. This suggests that the whistlerproducing lightning regions do not need to be centred upon the conjugate point. We are currently planning additional experimental measurements to try and better identify the Dunedin whistler source region.

\subsection{Automatic Scaling: Future Instrumentation}


An upgrade to the existing AWD systems is currently being developed, changing the existing systems to Automatic Whistler Detector and Analyser (AWDA) systems which will automatically scale the detected whistlers to derive plasma densities. The Dunedin-AWDA will be part of a network of AWDA systems, termed AWDAnet, currently being deployed around the world [Lichtenberger et al., 2007; 2008]. In addition, whistler-derived plasmaspheric electron number densities may be combined with observations of flux tube mass loadings measured with the cross-phase technique applied to ULF pulsation magnetometers [e.g., Grew et al., 2007] to monitor of the dynamical composition of the plasmasphere [e.g., Menk et al., 2004]. Initial studies combining AWD-whistlers with ULF cross-phase derived mass densities are currently underway [Heilig et al., 2008].

\section{Summary and Conclusions}

Single-hop lightning-generated whistlers are observed by ground-based VLF detectors after the electromagnetic energy has propagated through the source ionosphere, inside a field-aligned whistler duct in the plasmasphere, and finally through the ionosphere in the conjugate region. Whistlers can be used to determine plasmaspheric properties, including the plasmaspheric electron density and the $L$-shell of the whistler duct. In this paper we have examined the output of an Automatic Whistler Detector System operating in Dunedin, New Zealand. In the first 530 days of operation the AWD system captured 147,339 records, of which 61,448 contained whistlers, i.e., a system accuracy of $\sim 42 \%$. Over the period 20 May 2005 - 31 October 2006 the AWD recorded 92,528 individual whistler events containing 236,019 whistler traces. About 58\% of the Dunedin observed whistlers are made of a single trace, with multi-trace whistlers ranging from 2-33 traces per event. The average number of traces in a Dunedin whistler event is $\sim 2.6$ traces/event. This equates to a whistler event rate of 0.12 per minute, and a whistler trace rate of 0.31 per minute. Despite the conjugate lightning levels for Dunedin being 1500 lower than that 
for Hungary, the AWD-reported whistler rate from Dunedin was only 3 times lower. Dunedin whistler rates are high, hundreds of times higher than would be estimated from conjugate lightning activity alone, showing that conjugate lightning activity levels are not a good predictor of whistler rates. Dunedin-observed whistlers are most common during the day time, in contrast with earlier findings. North American lightning may be the principle source of Dunedinwhistlers, but this is still to be confirmed.

The Eötvös University Automatic Whistler Detector (AWD) based at Dunedin has detected a large population of whistlers over a wide range of $L$-shells and with a sufficiently small false trigger rate to allow rapid processing of the data. An Automatic Whistler Detector and Analyser system which is in the last stages of development may soon provide regular near-automatic plasmaspheric measurements to the wider scientific community.

Acknowledgments. C.J.R. would like to thank Colette Houliston of Whitchurch, England for her support. The authors would like to thank the NASA LIS/OTD Science Team and Global Hydrology and Climate Center (GHCC) for collecting and providing the satellite observed lightning data.

\section{References}

Al'pert, Ya. L., 40 years of whistlers, J. Atmos. Terr. Phys., 42, 1 - 20, 1980.

Barr, R., D. Ll. Jones, and C. J. Rodger, ELF and VLF Radio Waves, J. Atmos. Sol. Terr. Phys., 62(17-18), 1689-1718, 2000.

Borovsky, J. E., and M. H. Denton, A statistical look at plasmaspheric drainage plumes, J. Geophys. Res., 113, A09221, doi:10.1029/2007JA012994, 2008. 
Borovsky, J. E., and J. T. Steinberg, The "calm before the storm" in CIR/magnetosphere interactions: Occurrence statistics, solar-wind statistics, and magnetospheric preconditioning, J. Geophys. Res., 111, A07S10, doi:10.1029/2005JA011397, 2006.

Burgess, W.C., and Inan, U. S., The role of ducted whistlers in the precipitation loss and equilibrium flux of radiation belt electrons, J. Geophys. Res., 98, 15643-15665, 1993.

Chang, H. C., and U. S. Inan, Quasi-relativistic electron precipitation due to interactions with coherent VLF waves in the magnetosphere, J. Geophys. Res., 88, 318-328, 1983.

Christian, H. J., R. J. Blakeslee, D. J. Boccippio, W. L. Boeck, D. E. Buechler, K. T. Driscoll, S. J. Goodman, J. M. Hall, W. J. Koshak, D. M. Mach, and M. F. Stewart, Global frequency and distribution of lightning as observed from space by the Optical Transient Detector, J. Geophys. Res., 108(D1), 10.1029/2002JD002347, 2003.

Clilverd, M. A. and Thomson, N. R., Observation of two preferred propagation paths for whistler mode VLF signals received at a non-conjugate location, J. Atmos. Sol. Terr. Phys., 54, 1075$1080,1992$.

Clilverd, M. A., N. R. Thomson, and A. J. Smith, The influence of ionospheric absorption on mid-latitude whistler mode signal occurrence from VLF transmitters, J. Atmos. Terr. Phys., 55, 1469-1477, 1993.

Collier, A. B. , Hughes, A. R. W., Lichtenberger, J., and Steinbach, P., Seasonal and diurnal variation of lightning activity over southern Africa and correlation with European whistler observations, Annales Geophysicae, 529-542, 24, (2), 2006.

Grew R. S., F. W. Menk, M. A. Clilverd, B. R. Sandel, Mass and electron densities in the inner magnetosphere during a prolonged disturbed interval, Geophys. Res. Lett., 34, L02108, doi:10.1029/2006GL028254, 2007.

Heilig, B., P. Kovács, J. Lichtenberger, J. Reda, K. Pajunpää and T. Raita, Comparison of equatorial plasma mass density and electron density at $\mathrm{L}=1.85$ inferred from ULF and VLF 
measurements, 3rd VERSIM Workshop Book of Abstracts, ISBN 978-963-284-017-8 (paperback), Budapest, pg. 28, 2008.

Helliwell, R. A., Whistlers and related ionospheric phenomena, Stanford University Press, Stanford, California, 1965.

Lay, E. H., A. R. Jacobson, R. H. Holzworth, C. J. Rodger, and R. L. Dowden, Local time variation in land/ocean lightning count rates as measured by the World Wide Lightning Location Network, J. Geophys. Res., 112, D13111, doi:10.1029/2006JD007944, 2007.

Lichtenberger, J., Cs. Ferencz, L. Bodnar, D. Hamar, and P. Steinbach, Automatic whistler detector and analyzer (AWDA) system network: I. Automatic whistler detector, J. Geophys. Res., (in press), 2008.

Lichtenberger, J., C. Ferencz, L. Bodnar, A. R. W. Hughes, A. B. Collier, C. J. Rodger, and M. A. Clilverd, Automatic whistler detector and analyzer system network: A new tool for realtime monitoring of the plasmasphere, IUGG XXIV Scientific Program, Corriere dell Umbria, pg. 5, 2 June 2007.

Lyons, L. R., R. M. Thorne, and C. F. Kennel, Pitch-angle diffusion of radiation belt electrons within the plasmasphere, J. Geophys. Res., 77, 3455-3474, 1972.

McIlwain, C. E., Coordinates for mapping the distribution of magnetically trapped particles, J. Geophys. Res., 66, 11, 3681 - 3691, 1961.

Menk F. W., I. R. Mann, A. J. Smith, C. L. Waters, M. A. Clilverd, D. K. Milling, Monitoring the plasmapause using geomagnetic field line resonances, J. Geophys. Res., 109, A04216, doi:10.1029/2003JA010097, 2004.

Meredith, N. P., R. M. Thorne, R. B. Horne, D. Summers, B. J. Fraser, and R. R. Anderson (2003), Statistical analysis of relativistic electron energies for cyclotron resonance with EMIC waves observed on CRRES, J. Geophys. Res., 108(A6), 1250, doi:10.1029/2002JA009700. 
Park, C. G., Methods of Determining Electron Concentrations in the Magnetosphere from Nose Whistlers, J. Geophys. Res., 1972.

Rodger, C. J., M. A. Clilverd, N. R. Thomson, D. Nunn, and J. Lichtenberger, Lightning driven Inner radiation belt energy deposition into the atmosphere: Regional and Global Estimates, Annales Geophys., 23(11), 3419-3430, 2005.

Sazhin, S. S., M. Hayakawa, and K. Bullough, Whistler diagnostics of magnetospheric parameters: a review, Ann. Géophys., 10, 293 - 308, 1992.

Smith, A. J., M. B. Grieve, M. A. Clilverd, and C. J. Rodger, A Quantitative Estimate of the Ducted Whistler Power within the Outer Plasmasphere, J. Atmos. Sol.-Terr. Phys., 63, 61-74 2001.

Starks, M. J., R. A. Quinn, G. P. Ginet, J. M. Albert, G. S. Sales, B. W. Reinisch, and P. Song, Illumination of the plasmasphere by terrestrial very low frequency transmitters: Model validation, J. Geophys. Res., 113, A09320, doi:10.1029/2008JA013112, 2008.

Storey, L. R. O., An investigation of whistling atmospherics, Phil. Trans. Roy. Soc. (London), 246A, $113-141,1953$.

Tarcsai, G., Szemer'edy, P., and Hegymegi, L.: Average electron density profiles in the plasmasphere between $L=1.4$ and 3.2 deduced from whistlers, J. Atmos. Terr. Phys., 50, 607$611,1988$.

Thomson, N. R., M. A. Clilverd, and A. J. Smith, Evidence of more efficient whistler-mode transmission during periods of increased magnetic activity, Ann. Geophys., vol. 15(8), 9991004, 1997.

János Lichtenberger, Space Research Group, Eötvös University, PO Box 32, Budapest H 1518, Hungary. (spacerg@sas.elte.hu) 
Gregory McDowell, Craig J. Rodger and Neil R. Thomson, Department of Physics, University of Otago, P.O. Box 56, Dunedin, New Zealand. (email: mcdowell.greg@gmail.com , crodger@physics.otago.ac.nz,n_thomson@physics.otago.ac.nz).

RODGER ET AL.: AUTOMATIC WHISTLER DETECTION 


\section{FIGURE CAPTIONS}

Figure 1. The annual average global total lightning activity (in units of flashes $\mathrm{km}^{-2} \mathrm{yr}^{-1}$ ) transformed into CGM geomagnetic co-ordinates and mirrored around the geomagnetic equator. Dots mark receiving locations in New Zealand and Hungary, and their geomagnetic conjugates. The upper panel shows southern hemisphere lightning mirrored into the northern hemisphere, while the lower panel shows northern hemisphere lightning mirrored. [See the online version for the color version of this figure].

Figure 2. Example of a rather spectacular whistler captured by the Dunedin-based AWD during local nighttime conditions on 4 February 2006. [See the online version for the color version of this figure].

Figure 3. Variation in the number of traces observed in AWD-reported whistler events from Dunedin, New Zealand. Note that the $y$-axis is presented with a logarithmic scale.

Figure 4. Daily whistler trace rate at Dunedin as observed by the Automatic Whistler Detector. Also shown are the daily average Kp (heavy black line) and Dst (heavy red line) values. [See the online version for the color version of this figure].

Figure 5. Seasonal variation in Dunedin AWD-observed whistler traces.

Figure 6. Diurnal variation of AWD-reported whistler traces over the period 20 May 2005 - 31 October 2006. 


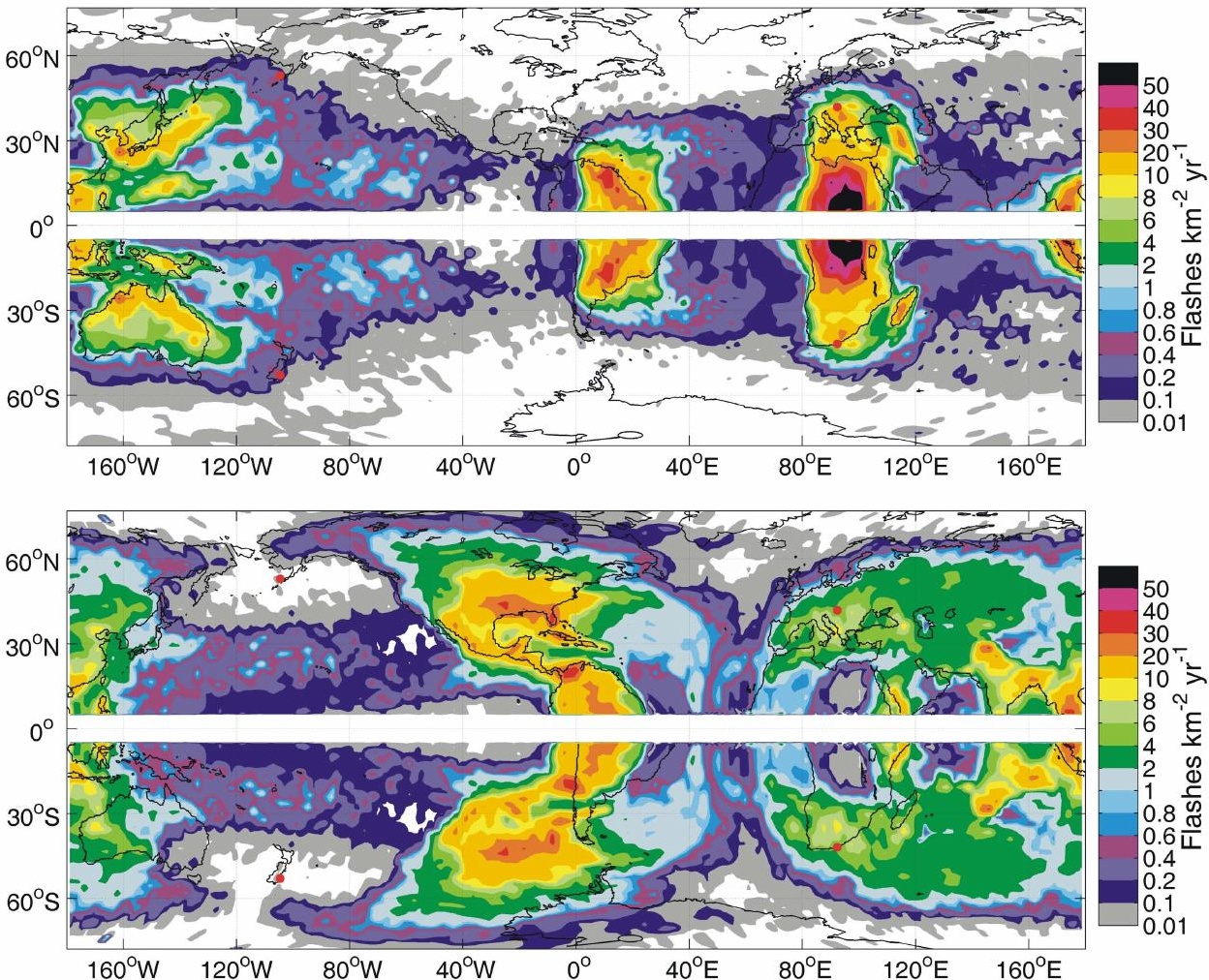




\section{Dunedin. 4 Feb 2006 11:50:24 UT}

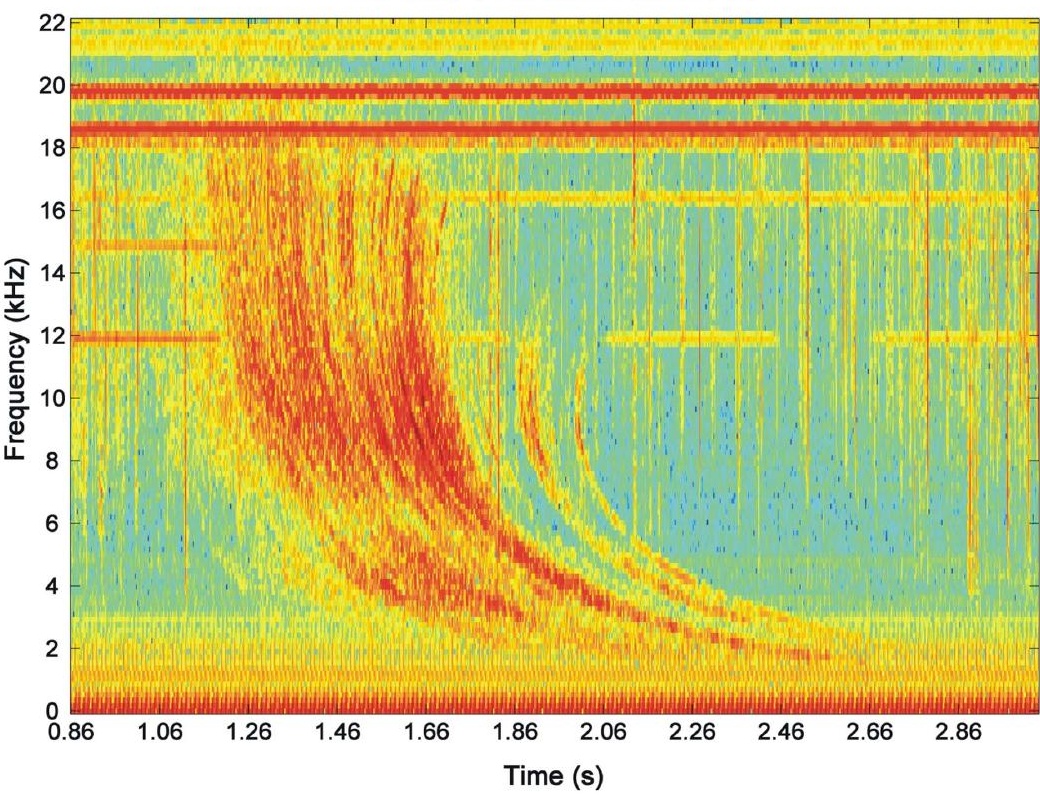




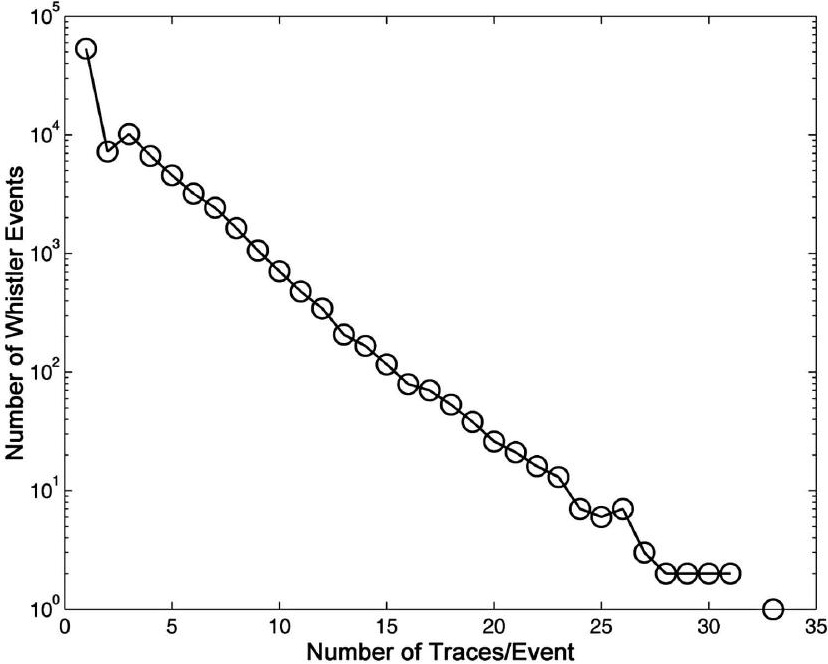




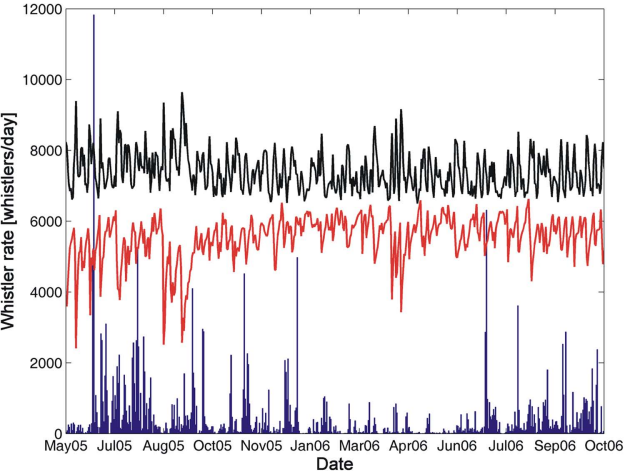




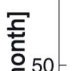

을 50

कั

(1)

닫

을

을 30 -

돈

$\Phi$

즌

(1) $20-$

U

(⿻)

ㄴ.

迹就 


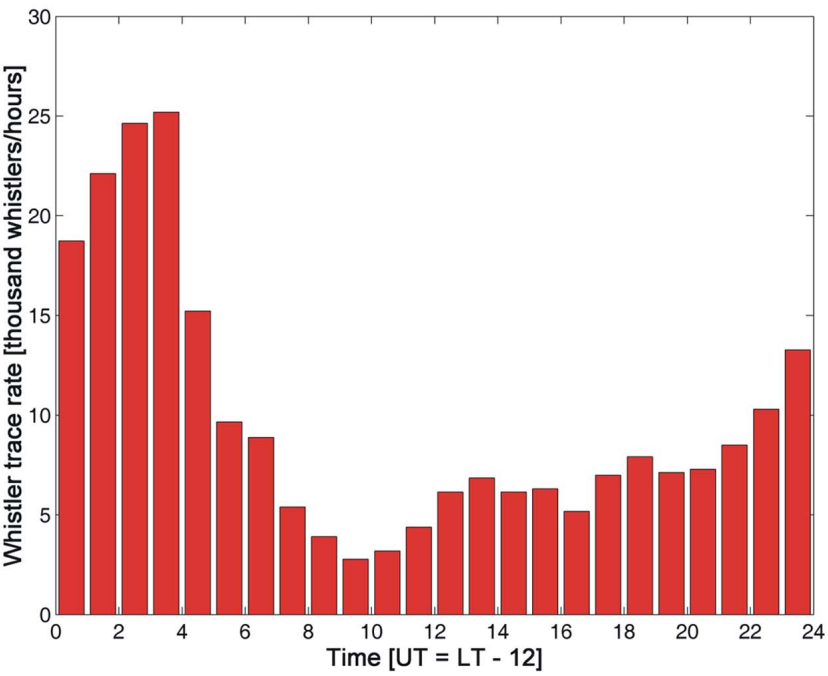

